\title{
Technical approach for the design of a high-resolution spectral model on a sphere: Application to decaying turbulence
}

\author{
K. Ishioka ${ }^{1}$, M. Yamada ${ }^{1}$, Y.-Y. Hayashi ${ }^{2}$, and S. Yoden ${ }^{3}$ \\ ${ }^{1}$ Graduate School of Mathematical Sciences, University of Tokyo. Tokyo. 153-8914. Japan \\ ${ }^{2}$ Division of Earth and Planetary Sciences. Hokkaido University. Sapporo 060-0810. Japan \\ ${ }^{3}$ Department of Geophysics, Kyoto University, Kyoto, 606-8502, Japan
}

Received: 21 June 1999 - Accepted: 18 November 1999

\begin{abstract}
Several technical suggestions to conslruct a highresolution spectral model on a sphere (the T682 barotropic model) are presented and their implementation in FORTRAN77 libraries is provided as a free software package ISPACK (http://www.gfd-dennou.org/arch/ispack/). A test experiment on decaying turbulence is conducted to demonstrate the ability of the model.
\end{abstract}

\section{Introduction}

Although the spherical harmonic spectral method is commonly used now in a wide range of numerical studies from Iwo-dimensional decaying turbulence on a sphere (Yoden and Yamada, 1993) to global climate modeling (Boer et al., 1992), the method is being abandoned in state of the art computing and other methods such as the spectral element method (Taylor et al., 1997), finite element method (Stuhne and Peltier, 1996), Fourier-based pseudospectral method (Fornberg and Merrill, 1997) and etc., are under development. This is because the spherical harmonic spectral method has a serious disadvantage when the method is used for high-resolution models. That is, the method requires the discrete spherical harmonic transform, but there is not any fast transform algorithms known currently for this case, which makes the method very costly compared with other new methods.

However, the spherical harmonic spectral method still has desirable features: it is easy to code and has complete isotropy. In particular, the latter feature is essential for theoretical studies, e.g., studies about turbulence on a sphere. Therefore, it is not meaningless to tune up spherical harmonic spectral models at high-resolution.

In this paper, we present not only several technical suggestions to construct a high-resolution spectral model on a sphere we also give a decaying turbulence experiment with the T682 barotropic model. We adopted the standard Eulerian formulation for its simplicity and its conservation prop- erly although the semi-Lagrangian formulation has been incorporated into state of the art numerical forcast models, e.g. Ritchie et al. (1995).

\section{Basic equations and spectral model}

The system under consideration is a non-divergent twodimensional flow with hyper viscosity on a rotating sphere. The flow is governed by the conservation law of the absolute vorticity $q(\lambda, \mu, t) \equiv \zeta+2 \Omega \mu$ following the fluid motion

$\frac{D q}{D t} \equiv \frac{\partial q}{\partial t}+F[\psi, q]=(-1)^{p+1} \nu_{2 p}\left(\nabla^{2}+2\right)^{p} q$,

$F[\psi, q] \equiv \frac{\partial \psi}{\partial \lambda} \frac{\partial q}{\partial \mu}-\frac{\partial \psi}{\partial \mu} \frac{\partial q}{\partial \lambda}$

where $\zeta(\lambda, \mu, t)$ is the vorticity $\left(\zeta \equiv \nabla^{2} \psi\right), \psi(\lambda, \mu, t)$ is the stream-function, $\lambda$ is the longitude, $\mu \equiv \sin \phi, \phi$ is the latitude, $t$ is the time, $\Omega$ is the angular speed of rotation of the sphere, $\nu_{2 p}$ is the hyper-viscosity coefficient, $p$ is the order of hyper-viscosity, and $\nabla^{2}$ is the horizontal Laplacian we have

$\nabla^{2} \equiv \frac{1}{1-\mu^{2}} \frac{\partial^{2}}{\partial \lambda^{2}}+\frac{\partial}{\partial \mu}\left\{\left(1-\mu^{2}\right) \frac{\partial}{\partial \mu}\right\}$.

Here the basic equation (1) is represented in dimensionless form.

Using the spectral method, we discretize the dependent variable $q$ using spherical harmonics as

$q(\lambda, \mu, t)=\sum_{n=1}^{M} \sum_{m=-n}^{n} \hat{q}_{n}^{m}(t) Y_{n}^{m}(\lambda, \mu)$

where, $M$ is the truncation number, and the $Y_{n}^{m}(\lambda, \mu)$ are spherical harmonics defined as

$Y_{n}^{m}(\lambda, \mu)=P_{n}^{m}(\mu) e^{\sqrt{1} m \lambda}$, 
where $P_{n}^{m}(\mu)$ is the associated Legendre functions defined as follows

$$
\begin{aligned}
P_{n}^{m}(\mu)= & \sqrt{(2 n+1) \frac{(n-|m|) !}{(n+|m|) !}} \frac{1}{2^{n} n !} \\
& \times\left(1-\mu^{2}\right)^{|m| / 2} \frac{d^{n+|m|}}{d \mu^{n+|m|}}\left(\mu^{2}-1\right)^{n} .
\end{aligned}
$$

Since $\hat{Y}_{n}^{m}$ is the eigenfunction of Laplacian as

$$
\begin{aligned}
\nabla^{2} Y_{n}^{m}= & -n(n+1) Y_{n}^{m}, \\
& (n=1,2, \cdots, M ; m=-n, \cdots, n),
\end{aligned}
$$

$\psi$ is calculated from $\hat{q}_{n}^{m}$ as follows

$\psi(\lambda, \mu, t)=\sum_{n=1}^{M} \sum_{m=-n}^{n} \hat{\psi}_{n}^{m}(t) Y_{n}^{m}(\lambda, \mu)$,

where,

$$
\begin{aligned}
\hat{\psi}_{n}^{m}= & -\frac{\hat{q}_{n}^{m}}{n(n+1)}+\frac{\Omega}{\sqrt{3}} \delta_{n 1} \delta_{m 0}, \\
& (n=1,2, \cdots, M ; m=-n, \cdots, n) .
\end{aligned}
$$

Multiplying the basic equation (1) by $Y_{n}^{-m}$, and integrating over the whole sphere yields the discretized ordinary differential equation

$$
\begin{aligned}
\frac{d \hat{q}_{n}^{m}}{d t}= & -\dot{F}_{n}^{m}-\nu_{2 p}\{n(n+1)-2\}^{p} \hat{q}_{n}^{m}, \\
& (n=1,2, \cdots, M ; m=-n, \cdots, n),
\end{aligned}
$$

where,

$$
\begin{aligned}
\hat{F}_{n}^{m}= & \frac{1}{4 \pi} \int_{0}^{2 \pi} \int_{-1}^{1} F[\dot{\psi}, q] Y_{n}^{-m} d \mu d \lambda, \\
& (n=1,2, \cdots, M ; m=-n, \cdots, n) .
\end{aligned}
$$

Here, the orthogonal relationship

$\frac{1}{4 \pi} \int_{0}^{2 \pi} \int_{-1}^{1} Y_{n}^{m} Y_{n^{\prime}}^{\prime m^{\prime}} d \mu d \lambda=\delta_{n n^{\prime}} \delta_{m m^{\prime}}$

is used.

Practically speaking, the integration (11) is calculated numerically as

$$
\begin{aligned}
\hat{F}_{n}^{m}= & \frac{1}{2 I} \sum_{i=1}^{I} \sum_{j=1}^{J} w_{j} F_{i j} Y_{n}^{-m}\left(\lambda_{i}, \mu_{j}\right) \\
& (n=1,2, \cdots, M ; m=-n, \cdots, n),
\end{aligned}
$$

where, $I$ and $J$ are the number of longitudinal and latitudinal grid points, respectively; $\lambda_{i}=2 \pi i / I, \quad(i=1,2, \cdots, I)$, $\mu_{j}\left(\mu_{1}<\mu_{2}<\cdots<\mu_{J}\right)$ are $J$ zero points of $\Gamma_{J}^{0}(\mu)$. Normally, the number of longitudinal and latitudinal divisions $I$ and $J$ are taken as $I>3 M$ and $J>3 M / 2$ lor de-aliasing. $F_{i j}$ is the value of $F[\ell, q]$ at the point $(\lambda, \mu)=\left(\lambda_{i}, \mu_{j}\right)$ calculated from

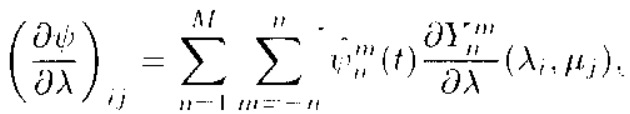

$\left(\frac{\partial \psi}{\partial \mu}\right)_{i j}=\sum_{n=1}^{M} \sum_{m=-n}^{n} \hat{\psi}_{n}^{m}(t) \frac{\partial Y_{n}^{m}}{\partial \mu}\left(\lambda_{i}, \mu_{j}\right)$,

$\left(\frac{\partial q}{\partial \lambda}\right)_{i j}=\sum_{n=1}^{M} \sum_{m=-n}^{n} \hat{q}_{n}^{m}(t) \frac{\partial Y_{n}^{m}}{\partial \lambda}\left(\lambda_{i}, \mu_{j}\right)$,

$\left(\frac{\partial q}{\partial \mu}\right)_{i j}=\sum_{n=1}^{M} \sum_{m=-n}^{n} \hat{q}_{n}^{m}(t) \frac{\partial Y_{n}^{m}}{\partial \mu}\left(\lambda_{i}, \mu_{j}\right)$,

as

$F[\psi, q] \equiv\left(\frac{\partial \psi}{\partial \lambda}\right)_{i j}\left(\frac{\partial q}{\partial \mu}\right)_{i j}-\left(\frac{\partial \psi}{\partial \mu}\right)_{i j}\left(\frac{\partial q}{\partial \lambda}\right)_{i j}$.

$w_{j}$ is the Gaussian weight defined as follows

$u_{j} \equiv \frac{2(2 n+1)\left(1-\mu_{j}^{2}\right)}{\left\{J P_{J-1}^{0}\left(\mu_{j}\right)\right\}^{2}}, \quad(j=1,2, \cdots, J)$.

\section{Technical suggestions for high-resolution model}

The costliest part of the spectral model introduced in the previous section is to evaluate the discrete transform (13) and the discrete inverse transforms (14)-(17). Since spherical harmonics consist of the associated Lengndre functions and trigonometric functions, these transforms can be divided into two transforms. The transform (13) is written

$$
\begin{aligned}
\hat{F}_{n}^{m}= & \frac{1}{2} \sum_{j=1}^{J} u_{j} \tilde{F}_{j}^{m} P_{n}^{m}\left(\mu_{j}\right), \\
& (n=1,2, \cdots, M ; m=-n, \cdots, n), \\
\tilde{F}_{j}^{m}= & \frac{1}{I} \sum_{i=1}^{I} F_{i j} e^{-\sqrt{-1} m \lambda_{i}}, \\
& (m=-M, \cdots, M ; j=1,2, \cdots, J),
\end{aligned}
$$

and inverse transforms (14)-(17) become

$$
\begin{aligned}
\left(\frac{\partial \mathcal{A}}{\partial \mu}\right)_{i j}= & \sum_{m=M}^{M}\left(\tilde{A}_{\mu}\right)_{j}^{m} e^{\sqrt{ }-1 m \lambda_{i}}, \\
\left(\frac{\partial A}{\partial \lambda}\right)_{i j}= & \sum_{m-M}^{M}\left(\tilde{A_{\lambda}}\right)_{j}^{m} e^{\sqrt{-1 m \lambda_{i}},} \\
& (i=1,2, \cdots, I ; j=1,2, \cdots, J), \\
\left(\tilde{A}_{\mu}\right)_{j}^{m} \equiv & \sum_{n=|m|}^{M} \hat{A}_{n}^{m} \frac{d P_{n}^{m}\left(\mu_{j}\right)}{d \mu}, \\
\left(\tilde{A}_{\lambda}\right)_{j}^{m} \equiv & \sqrt{-1} m \sum_{n=|m|}^{M} \hat{A}_{n}^{m} P_{n}^{m}\left(\mu_{j}\right), \\
& (j=1,2, \cdots, J ; m=-M, \cdots, M) .
\end{aligned}
$$

Here, A represents $q$ and $q$ in (14)-(17). The transforms (21) and (22) are nothing but Fourier transform, which can be calculated efficiently by using the FFT. For the translorms (20) 
and (2.3), however, there is no fast transform known except for the $m=0$ case, for which a fast approximate transform is devised by Alpert and Rokhlin (1991). Thus, the transforms (20) and (23) must be calculated straight forwardly. To calculate (20) by a computer, the main problem is CPU cost, but insufficiency of memory area becomes also a serious problem when the model resolution is very high. To store all the associated Legendre functions in memory, an array of size $J M(M+1) / 2$ is required. For the alias-free grid, this size is order of $3 M^{3} / 4$. In the test run, we set $M=682$, which makes the size of the array $2.38 \times 10^{8}$. In a double-precision calculation, this array requires $1.9 \mathrm{~GB}$ memory space. Although the required memory space as well as CPU cost can be made a half by utilizing symmetry of the associated Legendre functions, such a requirement makes the computation very difficult.

The suggestion to avoid this difficulty is very simple; the associated Legendre functions do not have to be stored if they are calculated every time the transforms (20) and (23) are calculated. This idea does not appear to be very sound for the following reasons.

1. The cost to compute the associated Legendre functions is of the same order as that to compute the transform. This appears to make the computation quitc costly.

2. The associated Legendre functions must be computed by the use of recurrence formulas. Such computations are not normally suitable for super computers with vector processors.

However, these two points do not lead to serious difficulty. Although the first point is perfectly true, it is possible to reduce the relative computational cost by executing several transforms simultaneously. For example, since $P_{n}^{-m}(\mu)=$ $P_{n}^{n t}(\mu)$, the Legendre transform for $\pm m$ can be executed simultaneously. Furthermore, the inverse Legendre transforms (23) for $\psi$ and $q$ should be computed together. The second point above is also true. However, this problem can also be avoided by setting the most inner do-loop variable to the latitude number $j$.

In the transform (23), not only the associated Legendre functions themselves but also their derivatives are required. However, the derivatives are commonly not used explicitly but the relationship

$$
\begin{aligned}
\left(1-\mu^{2}\right) \frac{d P_{n}^{m}}{d \mu} & =-n \sqrt{\frac{(n+1)^{2}-m n^{2}}{4(n+1)^{2}-1}} P_{n+1}^{m} \\
& +(n+1) \sqrt{\frac{n^{2}-m^{2}}{4 n^{2}-1}} P_{n-1}^{m}
\end{aligned}
$$

are substituted into the first equation to climinate the derivatives. We, instead, use the following recurrence formula

$$
\begin{aligned}
P_{n}^{m}= & \sqrt{\frac{2 n+1}{(2 n-1)(n+m)(n-m)}} \\
& \times\left\{n \mu P_{n-1}^{m}-\left(1-\mu^{2}\right) \frac{d P_{n-1}^{m}}{d \mu}\right\},
\end{aligned}
$$

$$
\begin{aligned}
& \left(1-\mu^{2}\right) \frac{d P_{n}^{m}}{d \mu} \\
& =-n \mu P_{n}^{m}+\sqrt{\frac{(2 n+1)(n+m)(n-m)}{2 n-1}} P_{n-1}^{m},
\end{aligned}
$$

to obtain $P_{n}^{m}$ and $d P_{n}^{m} / d \mu$ simultaneously. This does not reduce the amount of computation very much but makes the model program very simple.

Another difficulty comes from the hyper-viscosity term in (1). When the truncation number $M$ is very large, the coefficient in the second term on the right hand side of (10) becomes huge. This makes the ordinary differential equation (10) a "stiff" equation (see Press et al., 1992). Such a stiff equation is very difficult to integrate by explicit schemes, e.g., Runge-Kutta, because the time step must be kept very small to avoid numerical instability. The general solution to this problem is to use implicit schemes. However, implicit schemes, which require solving very large linear equations, are costlier than explicit schemes. To avoid this difficulty, we transformed the equation (10)) as

$$
\begin{array}{r}
\frac{d}{d t}\left(\hat{q}_{n}^{m} e^{\nu_{2 p}\{n(n+1)-2\}^{p} t}\right)=-\hat{F}_{n}^{m} e^{\nu_{2 p}\{n(n+1)-2\}^{p} t} \\
(n=1,2, \cdots, M ; m=-n, \cdots, n)
\end{array}
$$

and applied Runge-Kutta scheme to this transformed equation, which makes it possible to take larger time step.

These suggestions described above have becn already implemented as FORTRAN77 libraries in a free software ISPACK (Ishioka, 1999), which is available from http: // Www.gfd-dennou. org/arch/ispack/.

\section{Setup of the test experiment}

As a test run, we conducted a numerical experiment at similar setup as Yoden et al. (1999) except that only a non-rotating $(\Omega=0)$ case is considered in this paper. The basic equation (1) is integrated from an initial random flow field, of which energy spectrum is given by,

$E(n, t=0)=\frac{A n^{\gamma / 2}}{\left(n+n_{0}\right)^{\gamma}}, \quad(n=2, \cdots, M)$.

Here the energy density is given by,

$$
E(n, t) \equiv \frac{1}{2} n(n+1) \sum_{m=-n}^{m=n}\left|\hat{\psi}_{n}^{m}\right|^{2}, \quad(n=2, \cdots, M) .
$$

Here the complex amplitude is determined so that it has random amplitude and phase under the above restriction.

In (28), the constant $A$ is defined as follows

$$
A=\sum_{n-2}^{M} \frac{\left(n+n_{0}\right)^{\imath}}{n^{\gamma / 2}}
$$

so that the total kinetic energy

$\mathcal{E} \equiv \sum_{n-2}^{M} E(n, t=0)=1$. 
The computation of the basic equation (1) is conducted with the spectral method described in Sect. 2. The truncation wavenumber $M$ is set to 682 , and the order of hyper-viscosity $p$ is set to 8 with the coefficient $\nu_{2 p}=1 \times 10^{-43}$. The spectral peak of initial energy $n_{0}$ is set to 50 , and the parameter $\gamma$, which decides the initial energy distribution, is set to 100 . Time evolutions are computed with the fourth-order RungeKutta method applied to the transformed equation (27), in which the time increment $\Delta t$ is set to $5 \times 10^{-4}$.

\section{Results of the test experiment}

Firstly, we check the efficiency of the implementation of the model. Figure 1 shows the CPU-time required to integrate the model by one time-step on the FUJITSU VPP500 supercomputer for several truncation numbers $M$. Although the operation counts needed for one time-step integration are of order $M^{3}$, the CPU-time is approximately proportional to $M^{2}$ where $M$ is smaller than about 200 . This is because the length of the most inner do-loop, i.e., "vector length" in the implementation of the spherical harmonic transform is $J / 2 \sim 3 M / 4$, which enables the vector processor CPU to conduct operations fastcr in proportion to $M$. However, when $M$ is large enough, this acceleration saturates so that the CPU-time is proportional to $M^{3}$.

Secondly, we show some results of the test experiment described in the previous section. Figure 2 shows time evolution of energy spectrum $E(n, t)$. A clear power law close to $n^{-4}$ appears in the enstrophy cascading range $n \geq n_{0}(=$ $50)$, which was not obtained in the T341 experiment of Yoden, et al, Our T682 model, which ensures a wider cascading range, makes it possible to see the power law clearly. This power law is considered to be due to the discontinuity of the vorticity field at the edge of the coherent vortices described below.

The corresponding vorticity field is shown in Fig. 3. The initial random vorticity field (not shown) has developed into a number of coherent vortices through mergers of vortices (Fig. 3). To demonstrate the advantage of our high-resolution model, a part of Fig. 3 is magnified and shown in Fig. 4, where very fine structure of vorticity filaments around the coherent vortices are clearly seen. Furthermore, the resolution of the model is high enough to magnify a part of Fig. 4 again to make finer structure of the vorticity filaments more clear (Fig. 5).

\section{Summary}

Several technical suggestions to construct high-resolution spectral models on a sphere were presented. A test experiment was conducted with the T'682 barotropic model constructed by using these suggestions. The results demonstrated the ability of the model and showed that the suggestions are actually useful to construct a high-resolution spectral model. A clear power law close io $n^{1}$ of the energy spectrum is also confirmed thanks to the very high resolution.
Acknomledgements. Computations in Sect. S were conducted on VPP5(60) supercomputer at Data Processing Center. Kyoto University, and the GFDDKNNOU Library (SGKS Group, 1995) was used for drawing the figures. This work was supported in part by the Grant-in-Aid for Scientific Research of the Ministry of Education, Science, Sports and Culture of Japan and by the Grant-in-Aid for the Research for the Future Program "Computational Science and Engineering" of the Japan Society for the Promotion of Science.

\section{References}

Alpert, B. K. and Rokhlin, V., A fast algorithm for the evaluation of Legendre expansions. SIAM J. Sci. Stut. Comput., 12, 158-179. 1991.

Boer, G.J., Arpe, K., Blackburn, M., Déqué, M., Gates, W. L., Hart. T.L. Le Treut, H., Roeckner, F., Sheinin, D. A., Simmonds. I., Smith. R. N. B., Tokioka, T., Wetherald, R. T.. and Williamson, D., Some results from an intercomparison of the climates simulated by 14 atmospheric general circulation models. J. Gophys. Res., 97, 12771-12786, 1992.

Fornberg, B. and Merrill. D., Comparison of finite differcnce- and pseudospectral methods for convective flow over a sphere. (ieophys. Res. Let., $24,3245-3248,1997$.

Ishioka, K., ispack-0.4.1, ht.tp: / /www gfd dennou . org/arck1/ ispack / GFI) Dennou Club (in Japanese), 1999.

Press, W. H.,Hlannery, B. P., Teukolsky, S. A., and Vetterling, W. T., Numerical Recipes in FORTRAN: the art of scientific compung-2nd.ed, Cambridge University Press, 963pp, 1992.

Ritchie, H., Temperton, C., Simmons, A.. Hortal, M., Davies, T., Dent, D., and Hamrud. M., Implementation of the semi-Lagrangian method in a high-resolution version of the ECMWF forcast model. Mon. Wea. Rev., $123,489-514,1995$.

SGKS Group, DCL-5.1.. ht.tp: //www.gfd-dennou. org/ $1 \mathrm{ibrary} /$ da 1/, GFD-Dennou Club (in Japanese), 1995

Stuhne, G. R. and Peltier, W. R., Vottex Frosion and amalgamation in a new model of large scale flow on the sphere. $J$. Comput. Phys., I28, 58-81, 1996.

Taylor, M., Tribbia, I., and Iskandarani, M., The spectral element method for the shallow water equations on the sphere. J. Comput. Phys., 130, 92-108, 1997

Yoden, S. and Yamada, M., A numerical experiment on two-dimensional decaying turbulence on a rotating spherc. $J$. Afmos. Sci., 50, 631-64.3. 1993

Yoden, S., Ishioka, K., Hayashi, Y.-Y., and Yamada. M., A futther experiment on two-dimensional decaying turbulence on a rotating sphere. Nuovo Cimento C, to apper. 1999. 

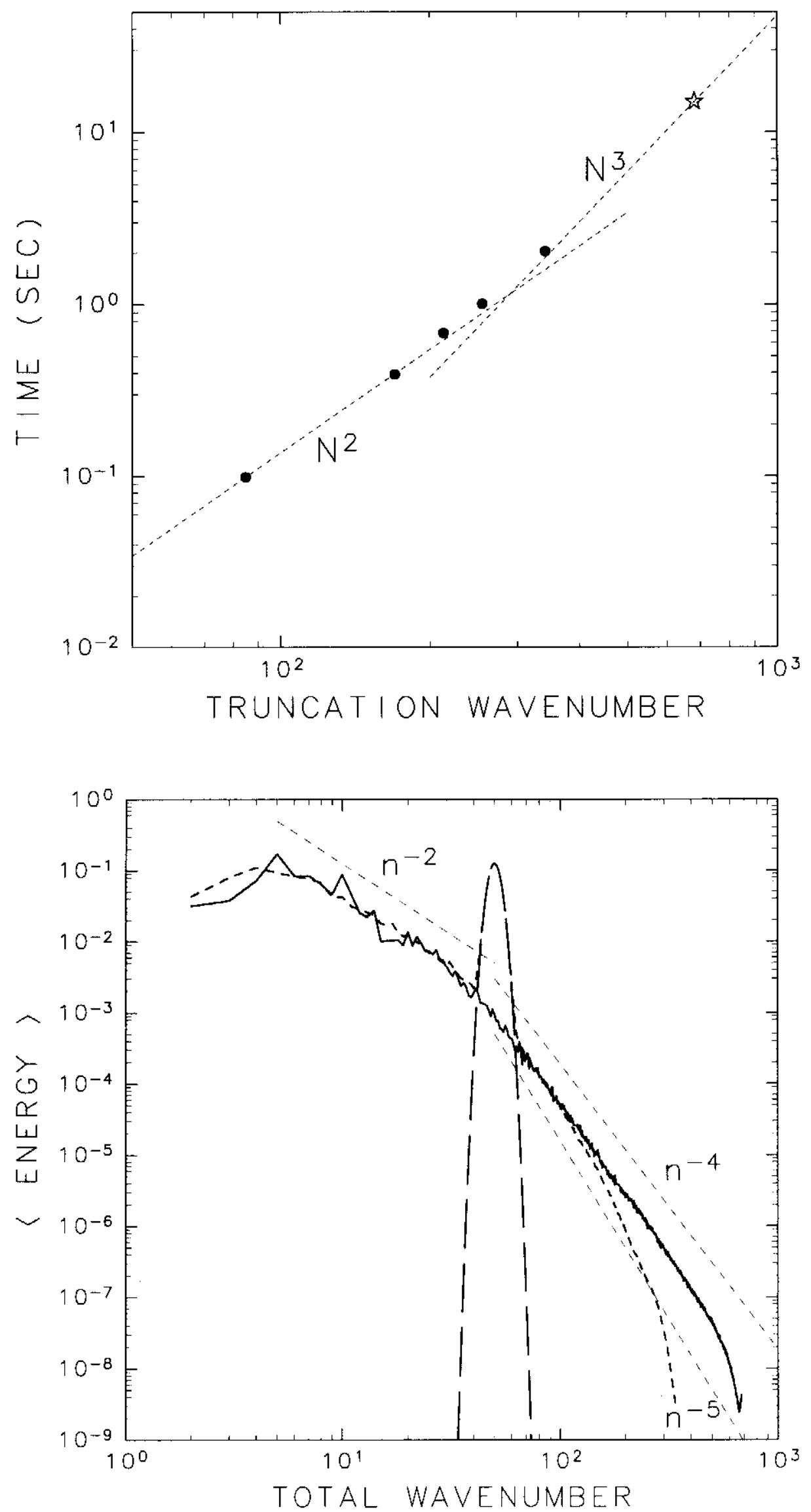

Fig. 1. C.PU time required to integrate the model by one lime-step on FUJITSU VPPS00 supercomputer for 6 cases of truncation number $M=85,170,213,255,341$, or 682 .

Fig. 2. Finergy spectrat at $t=0$ (broken line) and at $t=5$ (solid line). Dashed line is energy spectra at $t=5$ on T34l experiment adopted from Yoden et all. (1999) for comparison. 

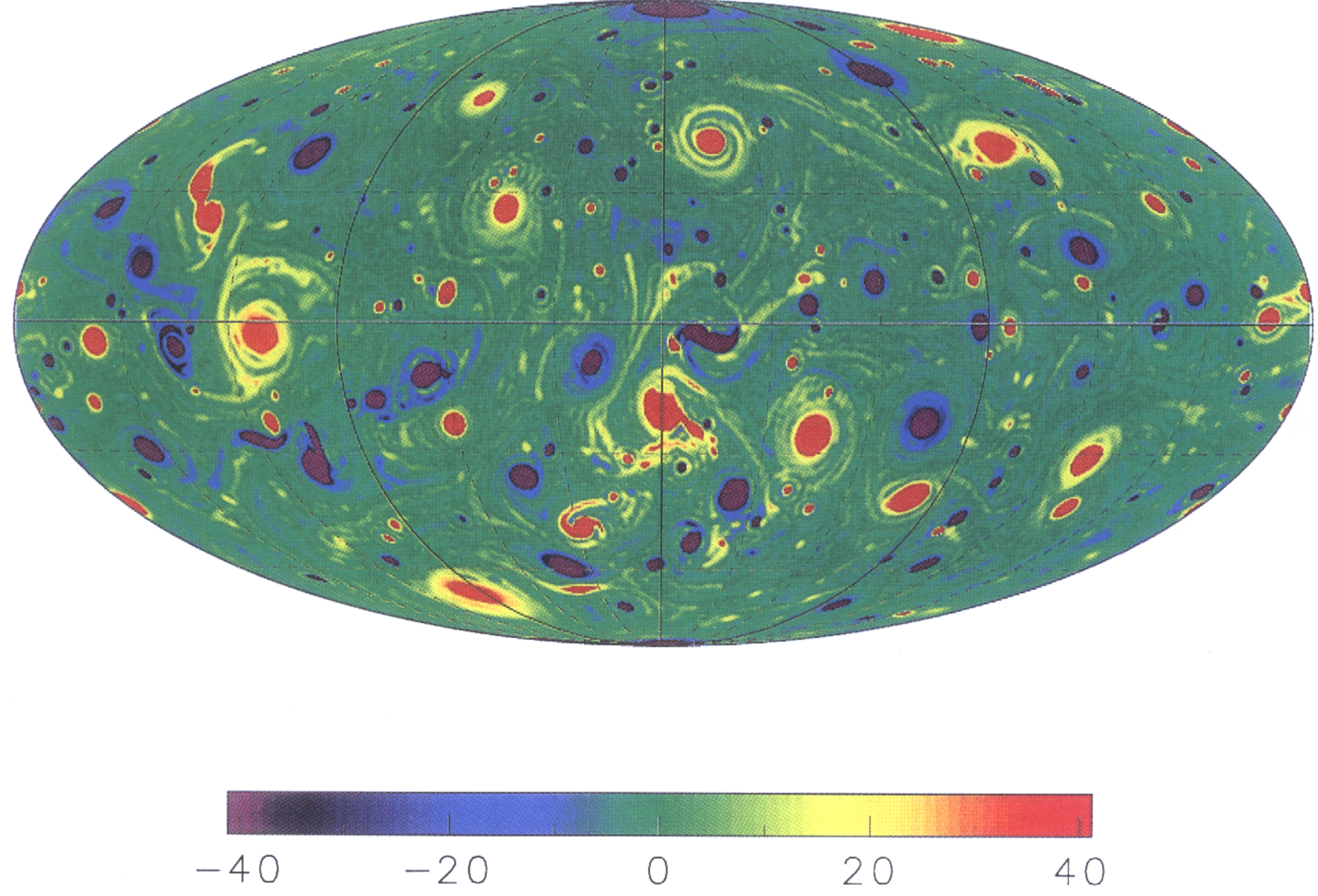

Fig. 3. Vorticity field at $t=5$. Whole sphere drawn with Mollweide projection centered at $(\lambda, \phi)=\left(90^{\circ}, 0^{\circ}\right)$.

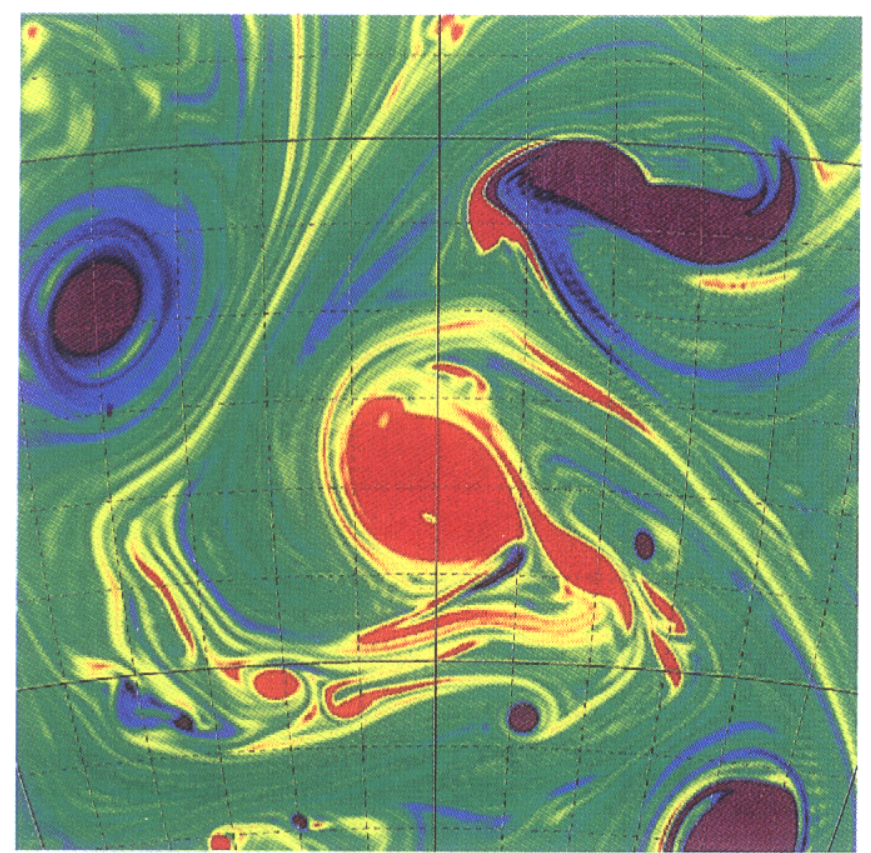

Fig. 4. Magnification of a part of Fig. 3 drawn with orthographic projection from $(\lambda, \phi)=\left(90^{\circ},-15^{\circ}\right)$. Lines of meridians and parallels are drawn for every $5^{\circ}$.

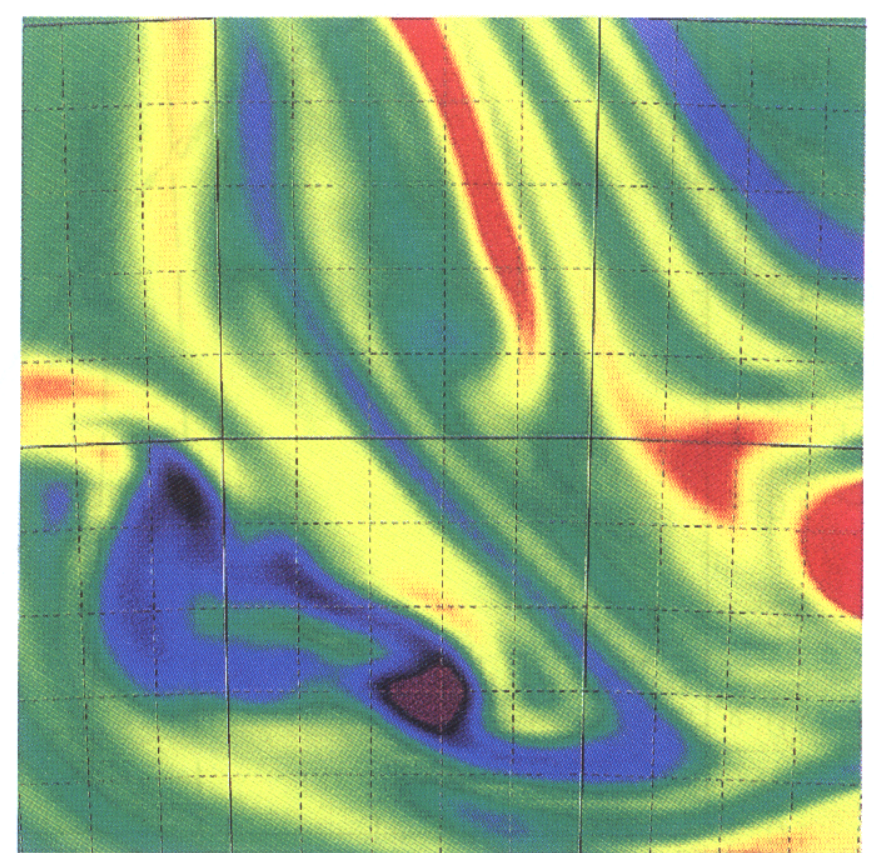

Fig. 5. Magnification of a part of Fig. 4 drawn with orthographic projection from $(\lambda, \phi)=\left(73^{\circ},-30^{\circ}\right)$. Lines of meridians and parallels are drawn for every $1^{\circ}$. 